\title{
A multiplexed vectored interface logic system for control of behavioral experiments
}

\author{
MICHAEL I. GAGE \\ U. S. Environmental Protection Agency, National Environmental Research Center, \\ Research Triangle Park, North Carolina 27711 \\ N. S. KENDRICK, JR. and J. T. CALLAHAN \\ School of Physics, Georgia Institute of Technology, Atlanta, Georgia 30332
}

\begin{abstract}
An interface was constructed which enabled a minicomputer to control operant conditioning experiments with a minimal amount of software recognition and input/output instructions. The hardware consisted of a real-time clock, logic to input a unique number with each change of state of digital input, and logic and solid state drivers to control standard, commercial $24 \mathrm{~V}$ behavioral stimuli.
\end{abstract}

A variety of interfaces have been described in print (e.g., Butler, 1974; Ciofalo, Tedford, Dodge, \& Goldberg, 1973; Kendrick, 1972; Millenson, 1973; Snapper \& Kadden, 1973) or are available from commercial suppliers (e.g., BRS/LVE; Grason-Stadler; Digital Equipment Corporation 1 ) that can transmit digital input and output information between small computers and devices in operant conditioning chambers. All of these interfaces require some sophisticated software to identify the input and select the appropriate processing routine. These identification routines usually take a considerable portion of real time, placing a limit on the number of experiments that can be controlled simultaneously by one computer.

An interface was designed with the primary purpose of reducing the time required to select the service routine appropriate for a given input through the extensive use of hardware in the form of rather inexpensive integrated circuits. The design is similar to one previously designed by Kendrick (1972) in that each input has a unique number that can be used as an address for selection of the service routine. However, the interface described here uses hardware running asynchronously with the computer to poll the inputs rather than the software interrogation used previously.

\section{HARDWARE CONFIGURATION}

The interface handles 60 inputs divided into groups of five inputs from each of 12 chambers. A 12-bit output buffer is connected to each of the chambers and four additional 12-bit buffers are available for controlling additional stimuli.

This project was funded by Contract Number 68-03-0329 with the U.S. Environmental Protection Agency. Reprints of this article and detailed schematic diagrams of the interface are available from Michael I. Gage, U. S. Environmental Protection Agency, NERC, EBL, Research Triangle Park, North Carolina 27711 .
The system is physically divided into two sections. The computer input and output connections and the interface logic components are mounted on module cards which plug into a mounting panel located in the cabinet that also contains the $\mathrm{PDP} / 8 \mathrm{E}$ computer (manufactured by Digital Equipment Corporation) used to control the experiments. The modules are organized to make maximum use of integrated circuit configurations. One module contains logic for decoding input/output pulses from the computer, the interrupt enable and skip circuitry, the logic for initialization of inputs, and the logic for scanning the input lines. A second module contains hardware for selecting the output buffer and clearing outputs. A third module is a real-time clock. There are four modules each containing circuits selecting and storing data from 16 inputs (with the last four inputs on one module reserved for use during initialization), and eight modules each containing circuits for two output buffers. Additional modules contain input and output cable terminations.

The input signal conditioners and output drivers are mounted in metal boxes located near each conditioning chamber, which may be as far as $20 \mathrm{~m}$ from the logic portion of the interface. Signals between the two sections of the interface are carried by 20 conductor ribbon cables, indicated by the lines with breaks in Figures 1 and 2, and consist only of the standard logic levels of 0 and $5 \mathrm{~V}$. Drivers in the remote metal boxes convert the output logic levels to the $24-\mathrm{V}$ signals required to control devices sold by most commercial behavioral testing equipment manufacturers. These devices are connected to the interface through screw terminal strips in the metal boxes to facilitate rapid replacement in case of component failure.

\section{INTERFACE LOGIC}

The interface logic consists of three parts: a real-time clock, input recognition circuits, and output buffer circuits. 


\section{Clock}

The clock contains a Type 555 resistor-capacitor oscillator and three scalers which divide the clock frequency. Frequencies of either $10 \mathrm{kHz}, 1 \mathrm{kHz}, 100 \mathrm{~Hz}$, or $10 \mathrm{~Hz}$ are selected by change of a jumper on the clock control board. The clock can be enabled to cause a computer interrupt at the selected frequency. Accumulated time is stored by incrementing a computer memory register.

\section{Input Circuits}

A simplified version of the logic involved to sense the status from one input device is shown in Figure 1. In the constructed interface, additional inverters, drivers, and logic elements exist which are not shown. Some integrated circuits are used as part of the input logic from more than one device. Inputs can be accepted either from devices that produce clean voltage level changes or from make after break switches (such as snap action switches) connected to flip-flop latches located in the remote boxes. The input line from the latch to the main logic modules stays at the high voltage level while contact with the input device is made, and remains at the low level when no contact is made with the device. However, only information about device change-of-state is transmitted to the computer because only a change in input line voltage sets off one of the two flip-flops associated with the device. The onset flip-flop is set when device contact is made and the offset flip-flop is set when device contact is broken.

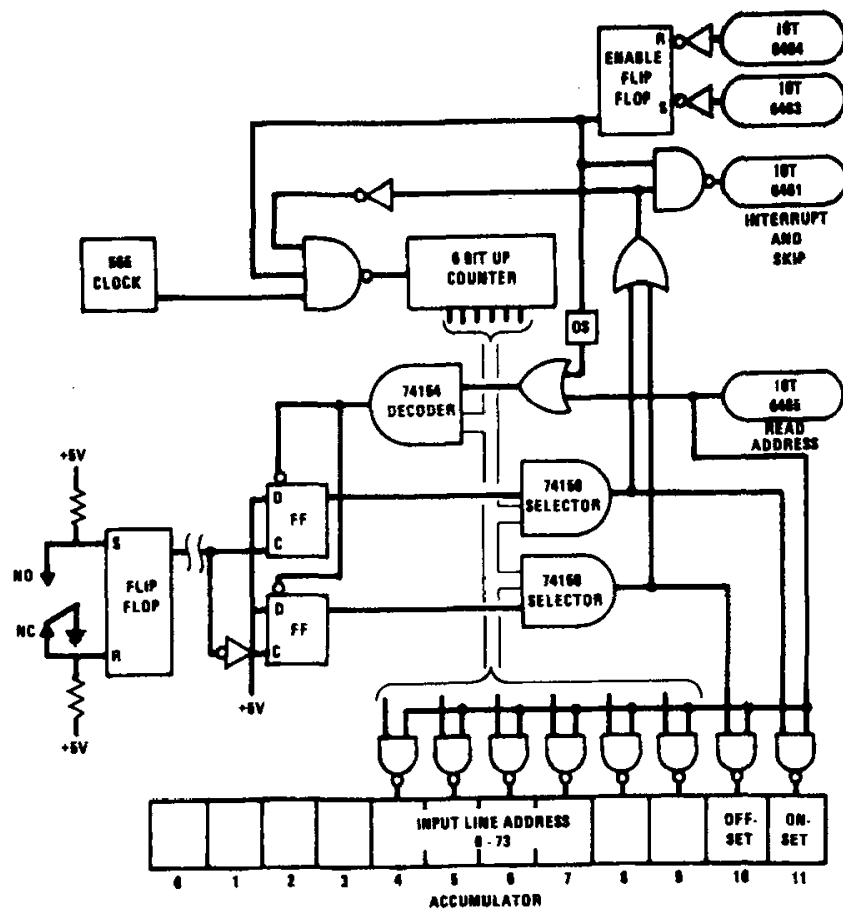

Figure 1. Simplified diagram of input interface from one device.
A 555 timer, different from the one used in the real-time clock, oscillating at a frequency of $850 \mathrm{kHz}$ is connected to a 6-bit binary up counter which is sequentially "and" gated to the set outputs of both flip-flops on the 60 input lines. When either the onset or offset flip-flop of the currently gated line is set, the clock is prevented from incrementing the counter, and signals are sent to the computer to cause a program interrupt. The states of the currently gated flip-flops and the counter value, which is the address of the gated input line, can then be read into the computer. Octal numbers $0-73$ are the input line addresses used. The onset and offset flip-flops are then cleared allowing the counter to resume incrementing, continuing the scan of input lines. When no flip-flops are set, a scan of all inputs takes approximately 75 microsec.

Additional circuitry, not shown in Figure 1, clears all onset and offset flip-flops during the first scan of the counter when it is initially enabled.

\section{Output Circuits}

The output logic is organized to allow control of up to 12 devices in each operant conditioning chamber through a separately addressable control buffer associated with each chamber. A simplified version of the logic required to address and control one of these buffers is shown in Figure 2. Some circuitry is left out of the figure and some of the diagrammed logic is used in control of more than a single buffer. An output control buffer is shown below the 12-bit register labeled accumulator. To change the state of any device, the address of the buffer controlling the device is first output from the computer to an output address buffer. In this interface, octal numbers from 0 to 17 are valid addresses. Then the entire contents of the addressed 12-bit control buffer are output. A logical 1 in a computer output register bit turns a device on and a logical 0 prevents the device from being turned on or tums it off. All output control buffers and the output address register can be cleared at one time through additional logic. This clearing feature is intended for use only during program initialization because it requires a high current drain.

In each of the 12 output control buffers connected to a conditioning chamber Bits 4-11 are connected through flip-flops to .25-A current drivers to control lamps or other stimuli that usually remain on for long periods of time. Bit 3 is connected through a flip-flop to a 2.5-A driver to control a stimulus requiring higher currents, such as a house light. Bits 0.2 are connected through one-shots to 2.5-A drivers to control feeders or other devices energized for rather short periods of time. Potentiometers on the logic modules are used to set the one-shots to the durations required by the device. One of the one-shots per buffer is connected to an inverter to control a device that requires a short lapse in energizing current, such as a water dipper. The use of one-shots 
eliminates the need for time consuming software routines to control many stimuli energized for short durations.

There are four additional 12-bit output control registers. Each bit in these registers is connected through a flip-flop to a .25-A current driver. These buffers are addressed in the same manner as the other control buffers and are numbered 14-17. They are not permanently assigned to any chambers but can be used wherever extra stimulus control is required.

\section{INSTRUCTION SET}

The input/output transfer instructions that allow a computer of the PDP $/ 8$ family to communicate with this interface are listed in Table 1 . In these machines, the input line address, output buffer address, and output buffer control contents are transferred between the computer and the interface buffers through the computer accumulator upon execution of the relevant input/output instructions. The interface is connected to the program interrupt facility so software service routines are entered only when an input event has occurred. The two skip instructions, 6451 and 6461, skip memory locations when the input is not from the interface. This saves instructions and time in a typical software polling of devices connected to the interrupt box.

The instruction 6463 enables the multiplexer and also initializes all inputs by clearing them with the first scan of the input lines. A single instruction, 6465 , is required to read the address and flip-flop status of the input line that has changed voltage level into the accumulator,

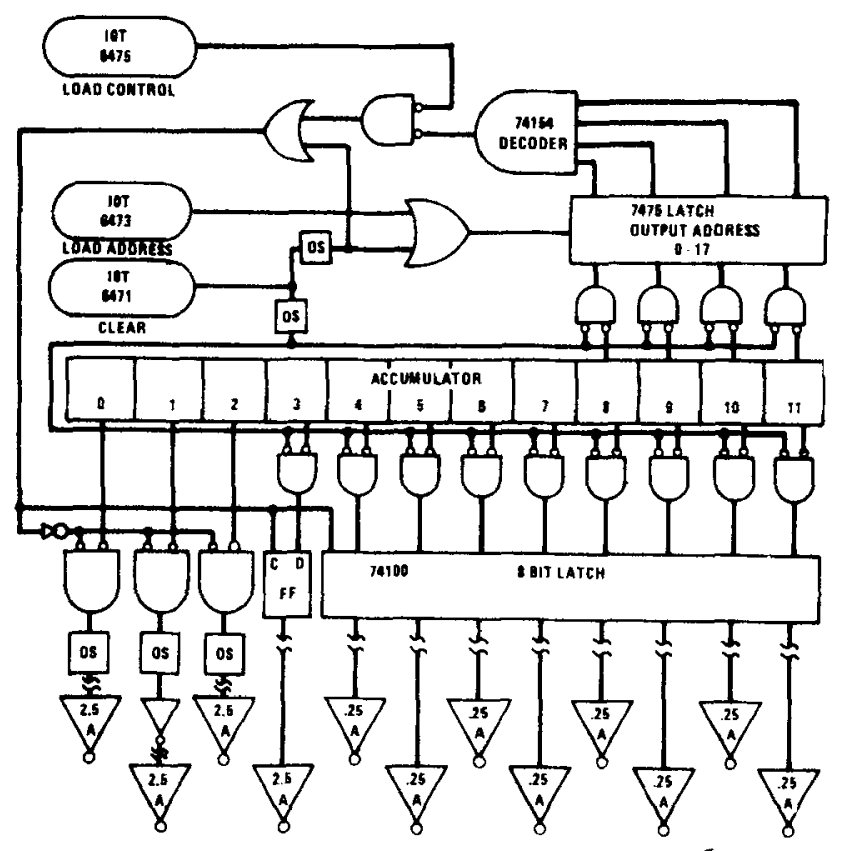

Figure 2. Simplified diagram of output interface to one experimental chamber.
Table 1

Input/Output Transfer Instructions Used for Interface

\begin{tabular}{cl}
$\begin{array}{l}\text { Device and } \\
\text { Instruction }\end{array}$ & liunction \\
\hline $\begin{array}{l}\text { Clock } \\
6451\end{array}$ & Skip if clock nag is not set \\
6452 & Clear flag and clear interrupt enable \\
6454 & Enable interrupt by clock \\
6456 & Microprogrammed 6452 and 6454 \\
Input & Skip if input flag is not set \\
6461 & Clear input interrupt flag \\
6462 & Start multiplexer scan, clear all input flip- \\
6463 & flops, enable interrupt by input \\
6464 & Clear interrupt enable \\
6465 & Read multiplexer address and input flip-flop \\
& status into accumulator, clear set input flip- \\
& flop, resume multiplexer scan
\end{tabular}

Output

6471 Clear all output control registers and address register

6473 Load output address register with accumulator

6475 Load addressed output control register with accumulator contents

reset both flip-flops, and continue the input counter scan. The input line address and flip-flop status can then be used as a vector to point directly to the starting location of the routine for servicing the input. In actual use, however, the onset flip-flop status bit is masked, and the resulting accumulator contents point to an address in a table which contains locations of data storage areas and locations of the start of service routines.

After execution of the output instructions 6473 and 6475 , the accumulator is not cleared so the current status of the output buffers may be stored in the computer memory. Only three device codes are used to address all the inputs and outputs connected to this interface. This leaves a large number of device codes available for interfacing additional peripherals to the computer.

\section{DISCUSSION}

This interface was built using a combination of integrated circuits on collage mounting boards, special printed circuit logic boards, and some modules and hardware obtained from Digital Equipment Corporation. However, the entire interface could be built from commercially available logic modules. The cost of design and construction of this system was about $\$ 8,000$, $\$ 3,000$ of which was spent for parts. The cost of components to interface each chamber was approximately $\$ 250$.

The interface was originally designed for connection to a negative bus PDP-8/I computer, but was modified to work with a positive input/output bus and on a PDP-8/E through the KA8-E Positive I/O Bus Interface option. By modifying the computer input and output sections this interface could probably work with any small computer. 
Expansion of the interface could be implemented by increasing the size of the input line scanning counter, output address buffer, and the number of input and output buffer modules.

During initial testing, a number of minor design changes were made to improve system function. These included amplifying and inverting some signals at several points in the interface and adding the inverters to several buffer one-shots. If a single bit was set or reset to indicate the status of the input onset and offset flip-flops, the input programming might be one instruction shorter. However, separate onset and offset status bits helped troubleshoot malfunctions on several occasions.

The interface has fulfilled the aims of the designers. It scans 60 input lines, each having two possible states, quickly enough to allow monitoring of rapidly occurring animal behavior such as licking at a water tube. The computer is interrupted from background tasks only when a change in input status occurs. The input counter scans each input line sequentially and resets only the gated line immediately after reading its address. This eliminates the possibility of reading a device address other than the one selected and also eliminates the possibility of missing an input state change while reading an address. Software recognition of the input line and jumping to the appropriate service routine can take as few as five instructions with a PDP-8. Since the interface scans inputs and controls outputs independently from the computer and with minimum program interaction, the computer can execute complex background programs in real time while still monitoring and controlling animal behavior.

\section{REFERENCES}

Butler, F. E. The BUS-ALL: An inexpensive set of interface for the SKED system. Behavior Research Methods \& Instrumentation, 1974, 6, 171-173.

Ciofalo, V. B., Tedford, R. H., Dodge, D. L., \& Goldberg, M. E. Application of a digital computer for research in psychopharmacology. In B. Weiss (Ed.), Digital computers in the behavioral laboratory. New York: Appleton-Century-Crofts, 1973.

Kendrick, N. S., Jr. Use of a PDP-8/L to train rats. DECUS Proce edings, 1972 (Spring), 233-243.

Millenson, J R. On-line sequential control of experiments by an automated contingency translator. In B. Weiss (Ed.), Digital computers in the behavioral laboratory. New York: Appleton-Century-Crofts, 1973.

Snapper, A. G. \& Kadden, R. M. Time-sharing in a small computer based on a behavioral notation sy stem. In $B$. Weiss (Ed.), Digital computere in the behavioral laboratory. New York: Appleton-Century-Crofts, 1973.

\section{NOTE}

1. Mention of manufacturer or cunnercial product name does not constitute endorsement or recommendation for use by the U. S. Environmental Protection Agency. 